\title{
NUCLEON SPIN STRUCTURE AND ITS CONNECTIONS TO VARIOUS SUM RULES
}

\author{
J. SOFFER \\ Centre de Physique Théorique, \\ UMR $6207{ }^{*}$ CNRS-Luminy Case 90\%, \\ 13288 Marseille Cedex 9, France \\ E-mail:soffer@cpt.univ-mrs.fr
}

\begin{abstract}
Our knowledge on the nucleon spin structure has greatly improved over the last twenty years or so, but still many fundamental questions remain unsolved. I will try to review some of the puzzling aspects of the origin of the nucleon spin. I will emphasize the connection with several sum rules and, when using this tool, the relevance of some kinematic regions for testing them in the QCD dynamics framework.
\end{abstract}

\section{Introduction}

The reply to the question " why do we need spin in high energy particle physics?", on very general grounds, is twofold: first we want to learn about hadron structure and second we want to test perturbative QCD in the spin sector. More specifically concerning the nucleon structure we need to determine the unpolarized parton distributions $f_{N}\left(x, Q^{2}\right)(N=p, n$ for proton and neutron), where $f$ stands for quarks $(u, d, s, .$.$) , antiquarks$ $(\bar{u}, \bar{d}, \bar{s}, .$.$) or gluons. We also need to know the corresponding helicity dis-$ tributions $\Delta f_{N}\left(x, Q^{2}\right)$ and transversity distributions. All these distributions are functions of the scaling variable $x$ and the $Q^{2}$ dependence, so the scaling violations predicted by $\mathrm{QCD}$, must be compared to experimental results. The data allow to extract various structure functions, unpolarized $F_{2}^{p, n}, F_{3}, .$. and polarized $g_{1}^{p, n}$, from deep inelastic scattering (DIS), which are expressed in terms of the parton distributions. The relevant experiments are currently performed at CERN (Compass), DESY (Hermes),

*UMR 6207 is Unité Mixte de Recherche du CNRS and of Universités Aix-Marseille I and Aix-Marseille II, and of Université du Sud Toulon-Var, Laboratoire affilié à la FRUMAM 
JeffLab and SLAC, but one should also mention the new RHIC spin programme at the polarized $p p$ collider at $\mathrm{BNL}^{1}$, which will go beyond testing the QCD scaling violations of the polarized parton distributions.

\section{Many sum rules on structure functions}

There exists a number of sum rules for unpolarized and polarized structure functions, some of which are rigorous results and other which rely on more or less well justified assumptions. Let us first consider the charged current structure functions in neutrino DIS, for which we have two rigourous results, namely the Adler sum rule ${ }^{2}$ (ASR)

$$
\int_{0}^{1} \frac{d x}{2 x}\left[F_{2}^{\bar{\nu} p}(x)-F_{2}^{\nu p}(x)\right]=N_{u}-N_{d}=1
$$

because

$$
N_{u}=\int_{0}^{1} d x u_{v a l}(x)=2 \text { and } N_{d}=\int_{0}^{1} d x d_{v a l}(x)=1,
$$

and the Gross-Llewellyn Smith sum rule ${ }^{3}$ (GLSSR)

$$
I_{G L S}=\int_{0}^{1} \frac{d x}{2 x}\left[x F_{3}^{\nu p}(x)+x F_{3}^{\bar{\nu} p}(x)\right]=N_{u}+N_{d}=3 .
$$

The ASR is exact and receives no QCD corrections, but its experimental verification is at a very low level of accuracy ${ }^{4}$. The GLSSR gets a negative QCD correction and the CCFR data ${ }^{5}$ gives $I_{G L S}=2.55 \pm 0.06 \pm 0.1$ at $Q^{2}=3 \mathrm{GeV}^{2}$, in fair agreement with the theoretical prediction.

Next, let us consider the unpolarized electromagnetic structure functions for proton and neutron $F_{2}^{p, n}(x)$, for which we have the Gottfried sum rule $^{6}$ (GSR). If one assumes an $\mathrm{SU}(2)$ symmetric sea, i.e. $\bar{u}(x)=\bar{d}(x)$, one can easily show, using Eq.(2), that

$$
I_{G}=\int_{0}^{1} \frac{d x}{x}\left[F_{2}^{p}(x)-F_{2}^{n}(x)\right]=\frac{1}{3} .
$$

In fact the NMC experiment ${ }^{7}$ has observed a large defect of the GSR, since their measurement gives at $Q^{2}=4 \mathrm{GeV}^{2}, I_{G}=0.235 \pm 0.026$. This flavor symmetry breaking, more precisely $\bar{d}>\bar{u}$, is a consequence of the Pauli exclusion principle which favors $d \bar{d}$ pairs with respect to $u \bar{u}$ pairs, since the proton contains two $u$ quarks and only one $d$ quark.

If we turn to polarized structure functions, there is first a fundamental result called the Bjorken sum rule $e^{8}$ (BSR). It was derived about thirty years ago in the framework of quark current algebra and it relates the first 
moment of the difference between $g_{1}^{p}(x)$ for the proton and $g_{1}^{n}(x)$ for the neutron and the neutron $\beta$-decay axial coupling

$$
\int_{0}^{1} d x\left[g_{1}^{p}(x)-g_{1}^{n}(x)\right]=\frac{1}{6} g_{A} / g_{V},
$$

where $g_{A} / g_{V}=1.2573 \pm 0.0028$ is very accurately known. The BSR gets also a negative QCD correction and we will come back later to the test of this firm prediction of QCD. One can also derive sum rules for $g_{1}^{p}$ and $g_{1}^{n}$ separately. These are the Ellis-Jaffe sum rules ${ }^{9}$ (EJSR) which read

$$
\begin{aligned}
& \Gamma_{1}^{p}=\int_{0}^{1} d x g_{1}^{p}(x)=\frac{1}{18}(9 F-D+6 \Delta s) \quad \text { and } \\
& \Gamma_{1}^{n}=\int_{0}^{1} d x g_{1}^{n}(x)=\frac{1}{18}(6 F-4 D+6 \Delta s),
\end{aligned}
$$

where $F=0.459 \pm 0.008$ and $D=0.798 \pm 0.008$ are the $\beta$-decay axial coupling constants of the baryon octet and $\Delta s=\int_{0}^{1} \Delta s(x) d x$ is the total polarization of the proton carried by the strange quarks. One recovers Eq. (5) by taking the difference because $F+D=g_{A} / g_{V}$. In their original work, Ellis and Jaffe made the critical assumption $\Delta s=0$, which allows to make definite predictions for $\Gamma_{1}^{p}$ and $\Gamma_{1}^{n}$. One gets $\Gamma_{1}^{p}=0.19$, in strong disagreement with the sixteen years old EMC data ${ }^{10} \Gamma_{1}^{p}=0.112 \pm 0.009 \pm 0.19$. This large defect, which still remains, was first attributed to a large $\Delta s$, but this naive interpretation has been ruled out since then, on experimental grounds. One should keep in mind that to test all the above sum rules, one requieres an accurate determination of the corresponding structure functions in the full kinematic range $0 \leq x \leq 1$, which is never achieved in current experiments. Significant progress will have to be achieved, to reduce the missing region, where some dangerous extrapolations are needed.

Concerning the other polarized structure function $g_{2}(x)$, which is related to transverse polarization, but has no simple interpretation in the parton model, it is possible to derive a superconvergence relation by considering the asymptotic behavior of a particular virtual Compton helicity amplitude. This leads to the Burkhardt-Cottingham sum rule ${ }^{11}$ (BCSR)

$$
\int_{0}^{1} d x g_{2}^{p}(x)=0 \text { and } \int_{0}^{1} d x g_{2}^{n}(x)=0,
$$

for proton and neutron and from this result, it has been naively argued that $g_{2}(x)$ vanishes identically. In fact, alternatively a simple relation between $g_{1}$ and $g_{2}$ can be expected, namely $g_{1}(x)+g_{2}(x)=0$. However $g_{2}(x)$ is more complicated than that ${ }^{12}$ and only part of it (its twist- 2 contribution) 
is entirely related to $g_{1}(x)$, by means of the Wandzura-Wilczek sum rule ${ }^{13}$ (WWSR) which reads for $J \geq 1$

$$
\int_{0}^{1} d x x^{J-1}\left[\frac{J-1}{J} g_{1}(x)+g_{2}^{W W}(x)\right]=0 .
$$

Clearly for $J=1$ one recovers the BCSR Eq. (7) and for $J=2$ one has

$$
g_{2}^{W W}(x)=-g_{1}(x)+\int_{x}^{1} g_{1}(y) \frac{d y}{y} .
$$

Finally, using the spin-dependent photoabsorption cross sections $\sigma_{1 / 2(3 / 2)}(\nu)$, Gerasimov-Drell-Hearn ${ }^{14}(\mathrm{GDH})$ have derived the following celebrated sum rule valid for real photons

$$
I_{G D H}=\int_{\nu_{t h r}}^{\infty}\left[\sigma_{1 / 2}(\nu)-\sigma_{3 / 2}(\nu)\right] \frac{d \nu}{\nu}=-\frac{2 \pi^{2} \alpha}{M^{2}} \kappa^{2},
$$

where $\nu$ is the photon energy in the target rest frame, $\nu_{t h r}$ is the pion production threshold, $\kappa$ the anomalous nucleon magnetic moment, $M$ the nucleon mass and $\alpha$ the fine structure coupling constant. However the GDH integral can be generalized to the case of absorption of polarized transverse virtual photons with $Q^{2}$

$$
I_{G D H}\left(Q^{2}\right)=\int_{\nu_{t h r}}^{\infty}\left[\sigma_{1 / 2}\left(\nu, Q^{2}\right)-\sigma_{3 / 2}\left(\nu, Q^{2}\right)\right] \frac{d \nu}{\nu} .
$$

One can show that, within a good approximation, one has in the scaling limit

$$
I_{G D H}\left(Q^{2}\right)=\frac{16 \pi^{2} \alpha}{Q^{2}} \Gamma_{1}
$$

where $\Gamma_{1}=\int_{0}^{1} d x g_{1}(x)$, so the GDH sum rule is connected to polarized DIS. The $Q^{2}$-dependence of the generalized GDH sum rule has been measured very accurately recently, with different targets, and we will come back in more details to some theoretical understanding of these data.

\section{The statistical approach for polarized parton distributions}

DIS of leptons on hadrons has been extensively studied, over the last twenty years or so, both theoretically and experimentally, to extract the polarized parton distributions of the nucleon. As it is well known, the unpolarized light quarks $(u, d)$ distributions are fairly well determined. Moreover, the data exhibit a clear evidence for a flavor-asymmetric light sea, i.e. $\bar{d}>\bar{u}$ 
as mentioned above, and large uncertainties still persist for the gluon $(G)$ and the heavy quarks $(s, c)$ distributions. The corresponding polarized gluon and $s$ quark distributions $(\Delta G, \Delta s)$ are badly constrained and we just begin to uncover a flavor asymmetry, for the corresponding polarized light sea, namely $\Delta \bar{u} \neq \Delta \bar{d}$. Whereas the signs of the polarized light quarks distributions are essentially well established, $\Delta u>0$ and $\Delta d<0$, this is not the case for $\Delta \bar{u}$ and $\Delta \bar{d}$. Here we briefly recall how we construct a complete set of polarized parton (all flavor quarks, antiquarks and gluon) distributions. Our motivation is to use the statistical approach ${ }^{15,16}$ to build up : $q_{i}, \Delta q_{i}, \bar{q}_{i}, \Delta \bar{q}_{i}, G$ and $\Delta G$, in terms of a very small number of free parameters. A flavor separation for the unpolarized and polarized light sea is automatically achieved in a way dictated by our approach.

The existence of the correlation, broader shape higher first moment, suggested by the Pauli principle, has inspired the introduction of FermiDirac (Bose-Einstein) functions for the quark (gluon) distributions ${ }^{17}$. After many years of research, we recently proposed ${ }^{15}$, at the input scale $Q_{0}^{2}=$ $4 \mathrm{GeV}^{2}$

$$
\begin{aligned}
x u^{+}\left(x, Q_{0}^{2}\right) & =\frac{A X_{0 u}^{+} x^{b}}{\exp \left[\left(x-X_{0 u}^{+}\right) / \bar{x}\right]+1}+\frac{\tilde{A} x^{\tilde{b}}}{\exp (x / \bar{x})+1}, \\
x \bar{u}^{-}\left(x, Q_{0}^{2}\right) & =\frac{\bar{A}\left(X_{0 u}^{+}\right)^{-1} x^{2 b}}{\exp \left[\left(x+X_{0 u}^{+}\right) / \bar{x}\right]+1}+\frac{\tilde{A} x^{\tilde{b}}}{\exp (x / \bar{x})+1}, \\
x G\left(x, Q_{0}^{2}\right) & =\frac{A_{G} x^{\tilde{b}+1}}{\exp (x / \bar{x})-1},
\end{aligned}
$$

and similar expressions for the other light quarks $\left(u^{-}, d^{+}\right.$and $\left.d^{-}\right)$and their antiparticles. We assumed $\Delta G\left(x, Q_{0}^{2}\right)=0$ and the strange quark distributions $s\left(x, Q_{0}^{2}\right)$ and $\Delta s\left(x, Q_{0}^{2}\right)$ are simply related ${ }^{15}$ to $\bar{q}\left(x, Q_{0}^{2}\right)$ and $\Delta \bar{q}\left(x, Q_{0}^{2}\right)$, for $q=u, d$. A peculiar aspect of this approach, is that it solves the problem of desentangling the $q$ and $\bar{q}$ contribution through the relationship 15

$$
X_{0 u}^{+}+X_{0 \bar{u}}^{-}=0
$$

and the corresponding one for the other light quarks and their antiparticles. It allows to get the $\bar{q}(x)$ and $\Delta \bar{q}(x)$ distributions from the ones for $q(x)$ and $\Delta q(x)$.

By performing a next-to-leading order QCD evolution of these parton distributions, we were able to obtain a good description of a large set of very 
precise data on $F_{2}^{p, n}\left(x, Q^{2}\right), x F_{3}^{\nu N}\left(x, Q^{2}\right)$ and $g_{1}^{p, d, n}\left(x, Q^{2}\right)$, in correspondance with eight free parameters ${ }^{15}$. Therefore crucial tests will be provided by measuring flavor and spin asymmetries for antiquarks, for which we expect ${ }^{15}$

$$
\begin{gathered}
\Delta \bar{u}(x)>0>\Delta \bar{d}(x), \\
\Delta \bar{u}(x)-\Delta \bar{d}(x) \simeq \bar{d}(x)-\bar{u}(x)>0 .
\end{gathered}
$$

For illustration, we show in Fig. 1 the predictions of the statistical approach with recent polarized DIS results.

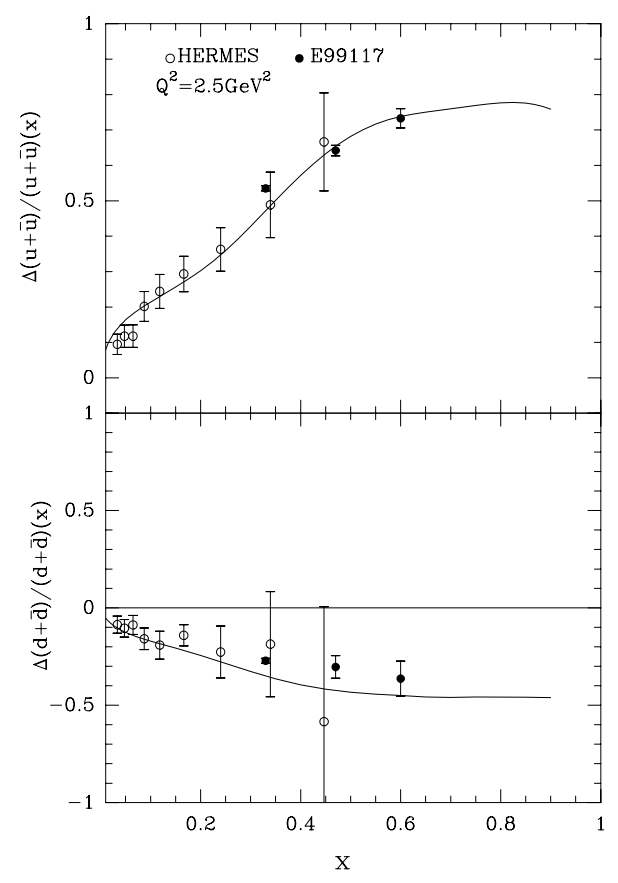

Figure 1. Results for $\Delta(u+\bar{u}) /(u+\bar{u})(x)$ and $\Delta(d+\bar{d}) /(d+\bar{d})(x)$ from Refs. [18,19], compared to the statistical model predictions.

The inequality $\bar{d}(x)-\bar{u}(x)>0$ has the right sign to agree with the defect in the GSR ${ }^{6}$. Let us make a few comments on the BSR. In the low 
$x$ region $(x \leq 0.1)$, both $g_{1}^{p}$ and $g_{1}^{n}$ are expected to increase, as well as their difference. The statistical prediction is close to the curve shown in Fig. 2, which has the simple expression $0.18\left(x^{-0.5}-1\right)$ implying 0.18 for the Bjorken integral. The statistical model gives the value 0.176 , in excellent agreement with the QCD prediction $0.182 \pm 0.005$ and with the world data $0.176 \pm 0.003 \pm 0.07^{20}$. We also note that if Eq. (18) above is satisfied, it means that the antiquark polarization contributes to the BSR. In the statistical model this contribution is 0.022 , which is not negligible. Finally this strong rise in the low $x$ region, which was first noticed in Ref. [21] is consistent with the results from the resummation of double-logarithmic contributions $^{22}$.

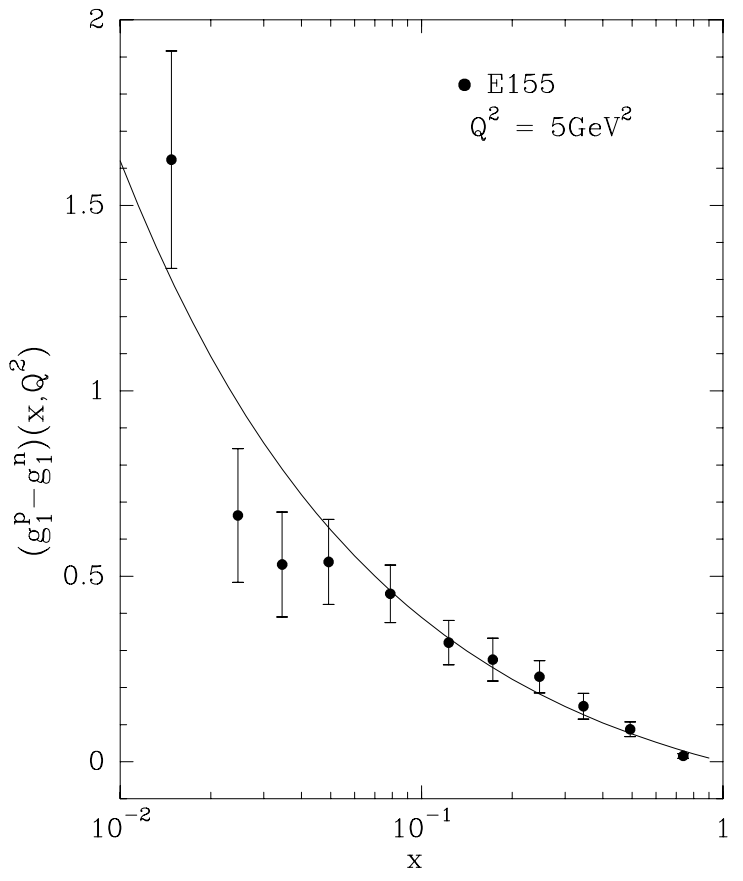

Figure 2. The data on $g_{1}^{p-n}\left(x, Q^{2}\right)$ from Ref. [20] compared to the curve given by $0.18\left(x^{-0.5}-1\right)$. 


\section{The generalized GDH sum rule revisited}

The generalized GDH sum rules ${ }^{14}$ are just being tested experimentally for proton, neutron and deuteron ${ }^{23,24,25,26}$. The characteristic feature of the proton data is the strong dependence on the four-momentum transfer $Q^{2}$, for $Q^{2}<1 \mathrm{GeV}^{2}$, with a zero crossing for $Q^{2} \sim 200-250 \mathrm{MeV}^{2}$, which is in complete agreement with our prediction ${ }^{27,28}$, published almost ten years ago. Our approach is making use of the relation to the BCSR for the structure function $g_{2}$, whose elastic contribution is the main source of a strong $Q^{2}$-dependence, while the contribution of the other structure function, $g_{T}=g_{1}+g_{2}$ is smooth. However, the recently published proton JeffLab data ${ }^{26}$ lie below the prediction, displaying quite a similar shape. Such a behaviour suggests, that the reason for the discrepancy may be the oversimplified treatment of the QCD expressions at the boundary point $Q_{0} \sim 1 \mathrm{GeV}$, defined in the smooth interpolation between large $Q^{2}$ and $Q^{2}=0$ and which serve as an input for our model. For large $Q^{2}$ we took the asymptotic value for the GDH integral and we neglected all the calculable corrections, as well as the contribution of the $g_{2}$ structure function. This was quite natural and unnecessary ten years ago, since no data was available at that time.

In a recent work ${ }^{29}$, we have filled up this gap by including the radiative (logarithmic) and power QCD corrections. The procedure used to take these corrections into account is explained in great details in Ref. [29]. We

found that the JeffLab data are quite sensitive to power corrections and may be used for the extraction of the relevant phenomenological parameters. In Fig. 3 we display for $\Gamma_{1}^{p}\left(Q^{2}\right)$, the comparison between the results of our previous work ${ }^{27,28}$ and the new analysis which leads to a curve fairly close to the JeffLab data ${ }^{26}$. We show in Fig. 4 the same comparison for $\Gamma_{1}^{n}\left(Q^{2}\right)$ and we notice that the strong oscillation around $Q^{2}=1 \mathrm{GeV}^{2}$, we had in the previous analysis, is no longer there. These results can be easily transformed into predictions for the Bjorken integral $\Gamma_{1}^{p-n}\left(Q^{2}\right)$ and for the deuteron $\Gamma_{1}^{d}\left(Q^{2}\right)$ which turn out to be in good agreement with preliminary JeffLab data ${ }^{30,31}$, in the low $Q^{2}$ region. 


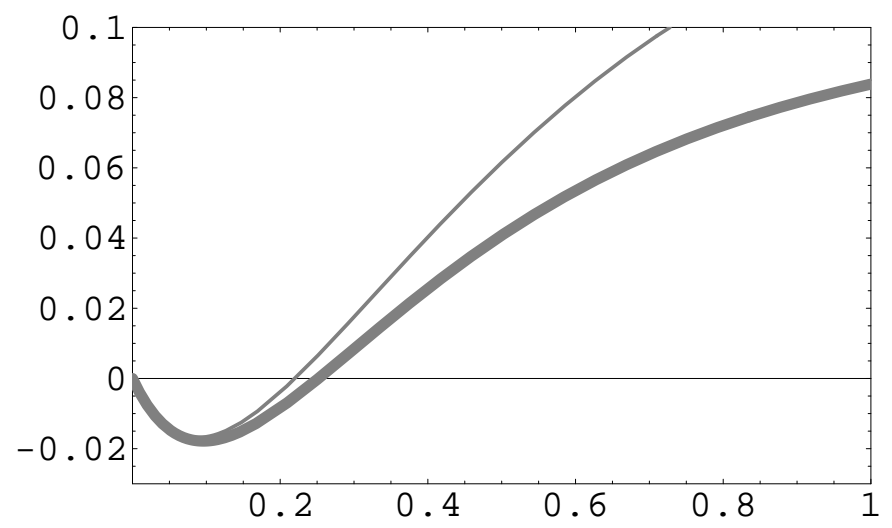

Figure 3. Our prediction for $\Gamma_{1}^{p}\left(Q^{2}\right)$. The thick line is the new analysis ${ }^{29}$, to be compared with the thin line, which represents our previous approach without corrections.

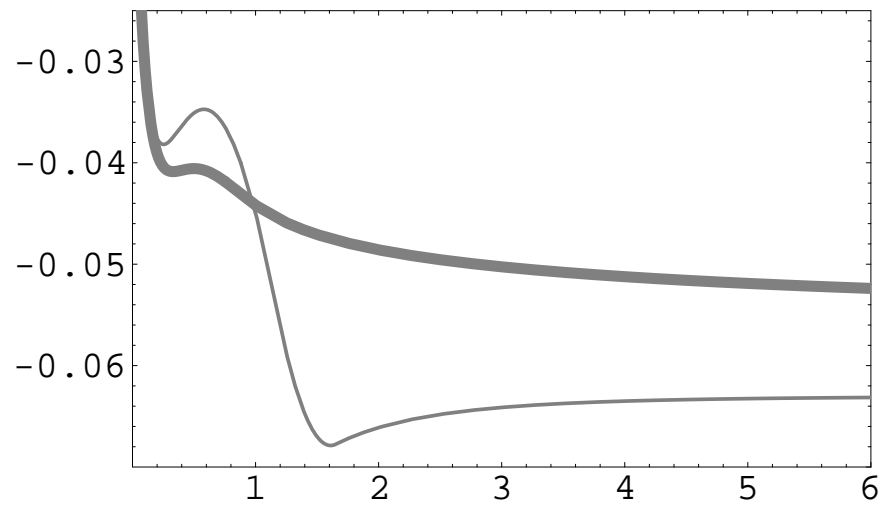

Figure 4. Our prediction for $\Gamma_{1}^{n}\left(Q^{2}\right)$. The thick line is the new analysis ${ }^{29}$, to be compared with the thin line, which represents our previous approach without corrections.

\section{Acknowledgments}

I would like to thank the organizers, in particular Sebastian Kuhn, for the invitation and for giving me the opportunity to deliver this talk at the very successful Symposium GDH2004. 


\section{References}

1. G. Bunce, N. Saito, J. Soffer and W. Vogelsang, Ann. Rev. Nucl. Part. Science 50, 525 (2000).

2. S. L. Adler, Phys. Rev. 143, 1144 (1966).

3. D. Gross and C. Llewellyn Smith, Nucl. Phys. B14, 337 (1969).

4. WA25 Collaboration, D. Allasia et al., Z. Phys C28, 321 (1985).

5. CCFR Collaboration, P. Z. Quintas et al., Phys. Rev. Lett. 71, 1307 (1993); W. C. Leung et al., Phys. Lett. B317, 655 (1993); J. H. Kim et al., Phys. Rev. Lett. 81, 3595 (1998).

6. K. Gottfried, Phys. Rev. Lett. 18, 1154 (1967).

7. New Muon Collaboration, M. Arneodo et al., Phys. Rev. D50, R1 (1994) and references therein; Phys. Lett. B364, 107 (1995).

8. J. D. Bjorken, Phys. Rev. 148 (1966), 1467 and ibid D1, 1376 (1970).

9. J. Ellis and R. Jaffe, Phys. Rev. D9, 1444 (1974); D10 1669 (E) (1974).

10. EMC Collaboration, J. Ashman et al., Phys. Lett. B206, 364 (1988).

11. H. Burkhardt and W. N. Cottingham, Ann. Phys. 56, 453 (1970).

12. X. Ji, Contribution to the Workshop on Deep Inelastic Scattering and QCD, Paris April 24-28, p. 435 (1995) (Editors J. F. Laporte and Y. Sirois).

13. S. Wandzura and F. Wilczek, Phys. Lett. B72, 195 (1977).

14. S. B. Gerasimov, Sov. J. Nucl. Phys. 2, 430 (1966); S. D. Drell and A. C. Hearn, Phys. Rev. Lett. 16, 908 (1966).

15. C. Bourrely, F. Buccella and J. Soffer, Eur. Phys. J. C23, 487 (2002). For a practical use of these PDF, see www.cpt.univ-mrs.fr/ bourrely /research/bbs-dir/bbs.html.

16. C. Bourrely, F. Buccella and J. Soffer, Mod. Phys. Lett. A18, 771 (2003).

17. C. Bourrely, F. Buccella, G. Miele, G. Migliore, J. Soffer and V. Tibullo, Z. Phys. C62, 431 (1994).

18. Hermes Collaboration, K. Ackerstaff et al., Phys. Lett. B464, 123 (1999).

19. Jlab E-99-117 Collaboration, X. Zheng et al., Phys. Rev. Lett. 92, 012004 (2004).

20. SLAC E155 Collaboration, P. L. Anthony et al., Phys. Lett. B493, 19 (2000).

21. J. Soffer and O. Teryaev, Phys. Rev. D56, 1549 (1997).

22. B.I. Ermolaev, M. Greco and S.I. Troyan, arXiv:hep-ph/0312029.

23. E143 Collaboration, K. Abe et al., Phys. Rev. Lett. 78, 815 (1997).

24. E94010 Collaboration, M. Amarian et al., Phys. Rev. Lett. 89, 242301 (2002).

25. HERMES Collaboration, A. Airapetian et al., Phys. Lett. B494, 1 (2000); Eur. Phys. J. C26, 527 (2003).

26. CLAS Collaboration, J. Yun et al., Phys. Rev. C67, 055204 (2003) and R. Fatemi et al., Phys. Rev. Lett. 91, 222002 (2003).

27. J. Soffer and O. Teryaev, Phys. Rev. Lett. 70, 3373 (1993).

28. J. Soffer and O. Teryaev, Phys. Rev. D51, 25 (1995).

29. J. Soffer and O. Teryaev, preprint CPT-2004/P.017.

30. A. Deur, these proceedings.

31. G. Dodge, these proceedings. 